EPJ Web of Conferences 61, 08008 (2013)

DOI: $10.1051 /$ epjconf/ 20136108008

(C) Owned by the authors, published by EDP Sciences, 2013

\title{
ALMA Continuum Spectrum of the M87 Nucleus
}

\section{Quasi-simultaneous continuum observations at bands-3, 6, 7, and 9}

\author{
Akihiro Doi ${ }^{1, a}$, Kazuhiro Hada ${ }^{2}$, Hiroshi Nagai ${ }^{3}$, Motoki Kino ${ }^{1}$, Mareki Honma ${ }^{3}$, Kazunori Akiyama ${ }^{4}$, Tomoaki \\ Oyama $^{3}$, and Yusuke Kono ${ }^{3}$ \\ ${ }^{1}$ The Institute of Space and Astronautical Science, Japan Aerospace Exploration Agency, 3-1-1 Yoshinodai, Chuou-ku, Sagami- \\ hara, Kanagawa 252-5210, Japan \\ ${ }^{2}$ INAF, Istituto di Radioastronomia, via Gobetti 101, Bologna I-40129, Italy \\ ${ }^{3}$ National Astronomical Observatory of Japan, 2-21-1 Osawa, Mitaka, Tokyo 181-8588, Japan \\ ${ }^{4}$ Department of Astronomy, Graduate School of Science, The University of Tokyo, 7-3-1 Hongo, Bunkyo-ku, Tokyo 113-0033, \\ Japan
}

\begin{abstract}
We report the preliminary result of ALMA continuum observations toward the M87 nucleus at thirtysix frequencies (quasi-simultaneously) ranging from $90 \mathrm{GHz}$ to $700 \mathrm{GHz}$. We detected the nuclear component at almost all the frequency points. We found a spectral break at the millimeter regime: a nearly flat spectrum at lower frequencies and a steep spectrum at higher frequencies. The spectral profile indicates a possible physical situation in the vicinity of the central supermassive black hole.
\end{abstract}

\section{Introduction}

M87 is one of the closest supermassive black holes forming relativistic jets beyond their host galaxy. Because of its proximity (16.7 Mpc; [1]) and a large estimated mass of the black hole $\left(\sim(3-6) \times 10^{9}\right.$ solar mass; e.g., [2]), M87 is one of the most promising target to be directly imaged toward the vicinity of the event horizon [3], as well as $\mathrm{Sgr} \mathrm{A}^{*}$ [4]. High-frequency very-long-baseline interferometry (VLBI) observations are required to see through surrounding environment around the jet launching region, due to optical depth effect and/or scatter broadening [5,6]. The highest frequencies that M87 has been VLBI-imaged so far are 43 and $86 \mathrm{GHz}$ (e.g., [7, 8, 11]). Compelling evidence of the collimation region down to $\sim 100$ Schwarzschild radii or less has been revealed on the basis of imaging analyses at up to these frequencies [9-11]. A VLBI visibility analysis at $230 \mathrm{GHz}$ for M87 also show a consistent result for the collimation of jets [3].

In this paper, we report the preliminary result of ALMA continuum observations toward the M87 nucleus at higher frequencies. Although the M87 nucleus has been observed over whole electromagnetic wavelengths so far (e.g., $[12,13])$, there has been no study over extensive frequency ranges at the millimeter-to-submillimeter regime. Our attempt aims to inspect the structure of jet starting point from the spectral frequency domain and to evaluate the prospect of future VLBI imagings of the innermost region of the M87 nucleus at higher frequencies.

\footnotetext{
ae-mail: akihiro.doi@vsop.isas.jaxa.jp
}

\section{Observations and Data Analyses}

We carried out ALMA observations in 2012 with twenty $12 \mathrm{~m}$-antenna array under the the extended configuration of 30-400 m baseline during Early Science Cycle 0. The precipitable water vapor (PWV) was $\sim 0.6 \mathrm{~mm}$ (a good condition) during the observations. Continuum observations were carried out with band-3, 6, 7, and 9 receivers. Four spectral windows, each of $1875-\mathrm{MHz}$ bandwidth, were used simultaneously; first local frequency switched sequentially to different nine frequencies. As a result, we employed 36 spectral windows over nearly whole available frequency range of the ALMA cycle-0 (90-700 GHz). The array configuration resulted in a synthesized beam of a range from to $2.6 \times 1.4 \operatorname{arcsec}^{2}$ to $0.29 \times 0.23 \operatorname{arcsec}^{2}$ at lowest and highest frequencies, respectively.

The data were reduced with the Common Astronomy Software Applications (CASA) package. We use a bright quasar for bandpass calibration and M87 itself for phase calibration. Data were integrated over four spectral windows for each three local settings at band-9, for sufficient signal-to-noise ratio. The absolute flux scale was established by observations of Titan. However, flux scaling factors determined using Titan possibly included different systematic errors among different spectral windows. Two radio sources, both of which show simple power-law spectra throughout ALMA bands, served as interband calibrators for this problem. The systematic errors can be estimated from the observed spectra of these calibrators, and can be corrected for the M87 spectrum.

All available Very Large Array (VLA) archival data obtained with A- or B-array configuration at multi- 

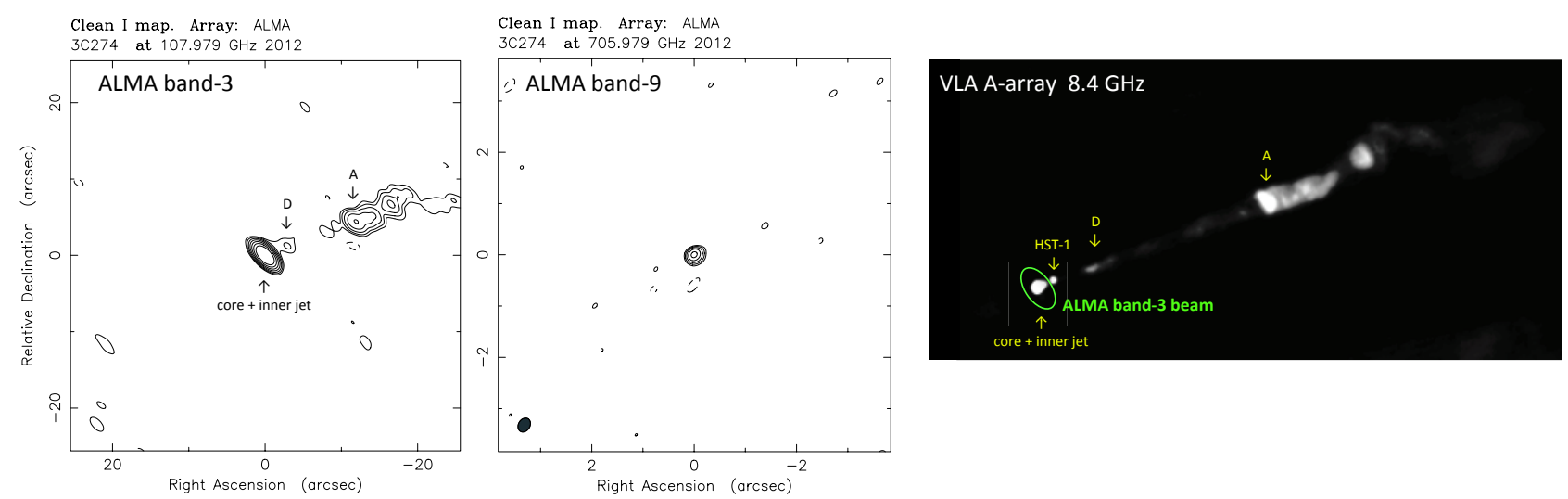

Figure 1. Left: ALMA image of the spectral window at a center frequency of $108 \mathrm{GHz}$ in band-3. Middle: ALMA image of the spectral window at a center frequency of $706 \mathrm{GHz}$ in band-9. Right: VLA image at $8.4 \mathrm{GHz}$. A ellipse represents the largest size of synthesized beams in this ALMA observations.

frequency bands (8.4-43 GHz) after 2006 were retrieved and analyzed for complementary spectra at the lowerfrequency range of radio regime.

\section{Results}

The ALMA image at band-3 show a similar radio structure to the VLA image, a bright component at the nucleus and kiloparsec-scale jets (see also a $230-\mathrm{GHz}$ continuum image from the Submillimeter Array (SMA); [14]). The largest ALMA beam involve core + inner jet contributions, whose structure can be partially resolved in the VLA image (Figure 1). Hence, the ALMA spectrum of the central component (not shown in this paper) can be interpreted as an upper limit for the core spectrum; VLBI core [11] contributes $\sim 40-60 \%$ and $\sim 70 \%$ in the VLA spectrum at centimeter and millimeter regimes, respectively. The contamination from the HST-1, which located $\sim 0.9$ arcsec away from the nucleus, can be practically negligible for the ALMA bands.

The VLA spectra are naturally connected with the ALMA spectrum at a spectral index of $\alpha \sim-0.2--0.3$ $\left(S_{v} \propto v^{\alpha}\right)$. It is noteworthy that a spectral break at approximately $200 \mathrm{GHz}$ is clearly detected in the ALMA spectrum. A steep spectrum of $\alpha \sim-1$ at $>200 \mathrm{GHz}$ proves that a flat-spectrum $(\alpha \sim 0)$ VLBI core disappears at the higher frequencies.

\section{Discussion}

The slightly flat spectra $(\alpha \sim-0.2--0.3)$ of VLA core + inner jet are presumably made of the sum of the flat spectrum of VLBA core and the steep spectrum of jet component. Therefore, the spectral break of the ALMA spectrum indicates that the core component cannot survive at the higher frequencies.

A core, a compact component at the apparent upstream end of jet structure in a VLBI image at a given frequency, is a photosphere whose surface is the site where the optical depth of synchrotron radiation is unity. The core location depends on the observing frequency ("core shift"; $[17,18])$. Multi-frequency astrometric VLBI observations at $2.3-43 \mathrm{GHz}$ confirm that the position of the core moves asymptotically toward the jet base with a $v^{-0.94}$ dependence for M87 [15]. Both of the frequency dependence in the core spectrum and the core shift are related to the radial profiles along a jet structure [16]. As long as the jet profile maintains, we cannot see the upper stream that should includes the innermost nuclear region around the black hole's event horizon.

Based on the spectral break in the ALMA spectrum, we can suggest a physical break of the jet profile in the vicinity of the black hole. The implications of this findings and the detailed descriptions of data analyses will be presented in a soon-to-be-submitted paper (Doi et al. in preparation). 


\section{References}

[1] Jordán, A., Côté, P., Blakeslee, J. P., et al. 2005, ApJ, 634, 1002

[2] Walsh, J. L., Barth, A. J., Ho, L. C., \& Sarzi, M. 2013, ApJ, 770, 86

[3] Doeleman, S. S., Fish, V. L., Schenck, D. E., et al. 2012, Science, 338, 355

[4] Doeleman, S. S., Weintroub, J., Rogers, A. E. E., et al. 2008, Nature, 455, 78

[5] Lo, K. Y., Backer, D. C., Ekers, R. D., et al. 1985, Nature, 315, 124

[6] Shen, Z.-Q., Lo, K. Y., Liang, M.-C., Ho, P. T. P., \& Zhao, J.-H. 2005, Nature, 438, 62

[7] Ly, C., Walker, R. C., \& Wrobel, J. M. 2004, AJ, 127, 119

[8] Krichbaum, T. P., Graham, D. A., Bremer, M., et al. 2006, Journal of Physics Conference Series, 54, 328
[9] Asada, K., \& Nakamura, M. 2012, ApJL, 745, L28

[10] Nakamura, M., \& Asada, K. 2013, arXiv:1308.1436

[11] Hada, K., Kino, M., Doi, A., et al. 2013, ApJ, 775, 70

[12] Perlman, E. S., Biretta, J. A., Sparks, W. B., Macchetto, F. D., \& Leahy, J. P. 2001, ApJ, 551, 206

[13] Doi, A., Kameno, S., Kohno, K., Nakanishi, K., \& Inoue, M. 2005, MNRAS, 363, 692

[14] Tan, J. C., Beuther, H., Walter, F., \& Blackman, E. G. 2008, ApJ, 689, 775

[15] Hada, K., Doi, A., Kino, M., et al. 2011, Nature, 477, 185

[16] Blandford, R. D., Konigl, A. 1979, ApJ, 232, 34

[17] Lobanov, A. P. 1998, A\&A, 330, 79

[18] Kovalev, Y. Y., Lobanov, A. P., Pushkarev, A. B., \& Zensus, J. A. 2008, A\&A, 483, 759 